\title{
Las hemerotecas digitales de la prensa en internet
}

\author{
Por Àngels Jiménez, Alfons González y M. Eulàlia Fuentes i Pujol
}

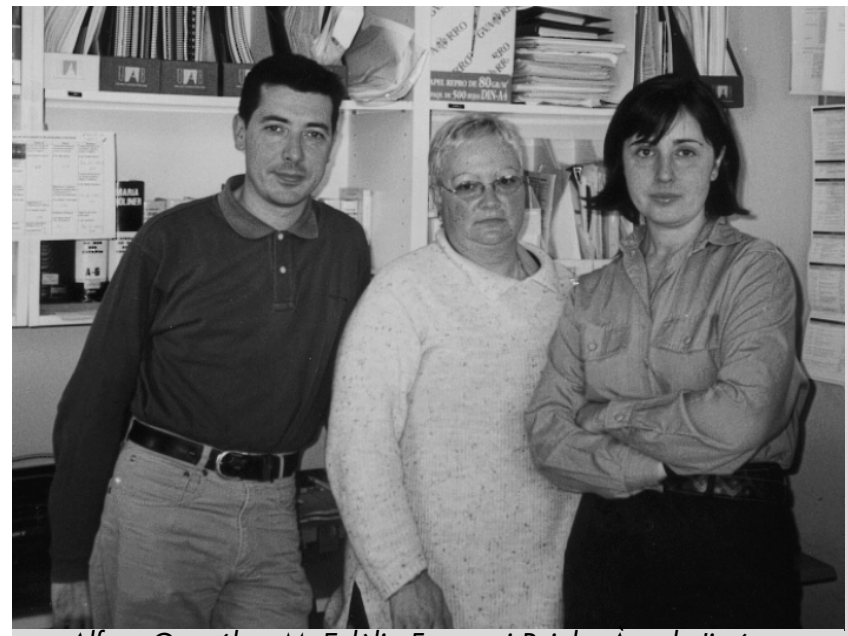

Alfons González, M. Eulàlia Fuentes i Pujol y Angels Jiménez
Resumen: El presente artículo analiza las características de las hemerotecas digitales de diarios nacionales e internacionales que, además de versión impresa, cuentan con edición en internet. Para el estudio se han observado numerosos diarios de todos los continentes, lo que ha permitido destacar aquellos que poseen las mejores hemerotecas y describir diversos aspectos relativos a las mismas: sistemas de acceso a la información retrospectiva, tipos de información accesible, especificidades de sus lenguajes de interrogación, opciones disponibles de visualización de resultados, así como ayudas ofrecidas al lector para optimizar la consulta. Se concluye ofreciendo una panorámica sobre la situación actual de las hemerotecas digitales así como de las principales tendencias que se observan en su evolución. Se proporcionan numerosos datos en forma de tablas y profusión de ejemplos relativos tanto a la prensa internacional como nacional.

Palabras clave: Hemerotecas digitales, Prensa en internet, Diarios digitales, Bases de datos de noticias, Recuperación de información, Almacenamiento de información, Información de actualidad.

\section{Title: Digital newspaper libraries on the internet}

Abstract: This article analyses the features of digital newspaper libraries of the national and international press services which have an internet edition besides the printed version. To carry out this survey, numerous newspapers from all over the world were studied. This has made it possible to highlight those with the best newspaper libraries and to describe different aspects related to them: system provided for accessing retrospective information, types of information available, details regarding the query languages, and options available for viewing search results, as well as the help offered to readers in order to maximize the results obtained. The article concludes with an overview of the current situation of digital newspaper libraries as well as of the main trends observed as they evolve. A variety of data are provided through charts and numerous examples taken from both the international and Spanish press.

Keywords: Digital newspaper libraries, Press services on the internet, On line newspapers, News databases, Information retrieval, Information storage, Current information.

Jiménez, Àngels; González, Alfons; Fuentes i Pujol, M. Eulàlia. "Las hemerotecas digitales de la prensa en internet". En: El profesional de la información, 2000, mayo, v. 9, n. 5, pp. 15-22.

\section{Introducción}

Cuando nació la televisión, muchos estudiosos creyeron y argumentaron que la radio perdería su hegemonía y sería sustituida por este nuevo medio de comunicación. Como se ha podido comprobar a lo largo de todos estos años, la televisión ha conquistado un espacio, pero no en detrimento de la radio. Los dos medios han coexistido y se han complementado.

En un sentido parecido el gran auge de internet, y concretamente la aparición de la world wide web, ha permitido la llegada de los medios de comunicación a la Red y muy especialmente la prensa digital o electrónica. En un primer momento fue, y en algunos casos aún sigue siendo, sólo un complemento de la publicada en formato papel, la reproducción facsímil del propio rotativo o también, en otros casos, una forma de publicidad e incluso un indicador de calidad.

Pero cada vez más grupos editoriales o más periódicos han empezado a ofrecer una serie de utilidades de valor añadido que permiten la mejora sustancial del propio producto y que la prensa tradicional no puede ni siquiera contemplar. Son servicios en los que se utilizan las técnicas y la gestión documentales. La documentación pasa a tener un sentido mucho más amplio, una aplicación más inmediata, más tangible. Se unen distintos ámbitos del saber: la comunicación, la información, la informática, las telecomunicaciones y la propia documentación.

\section{«Cada vez más periódicos han empezado a ofrecer una serie de utilidades de valor añadido que mejoran sustancialmente el propio producto»}

La prensa electrónica permite el acceso a grandes volúmenes de información, (retrospectiva, pero también actual) y para ello deben construirse sistemas de acceso y recuperación de la información de cualquier tipo, más o menos sofisticados, para dar respuesta a las 


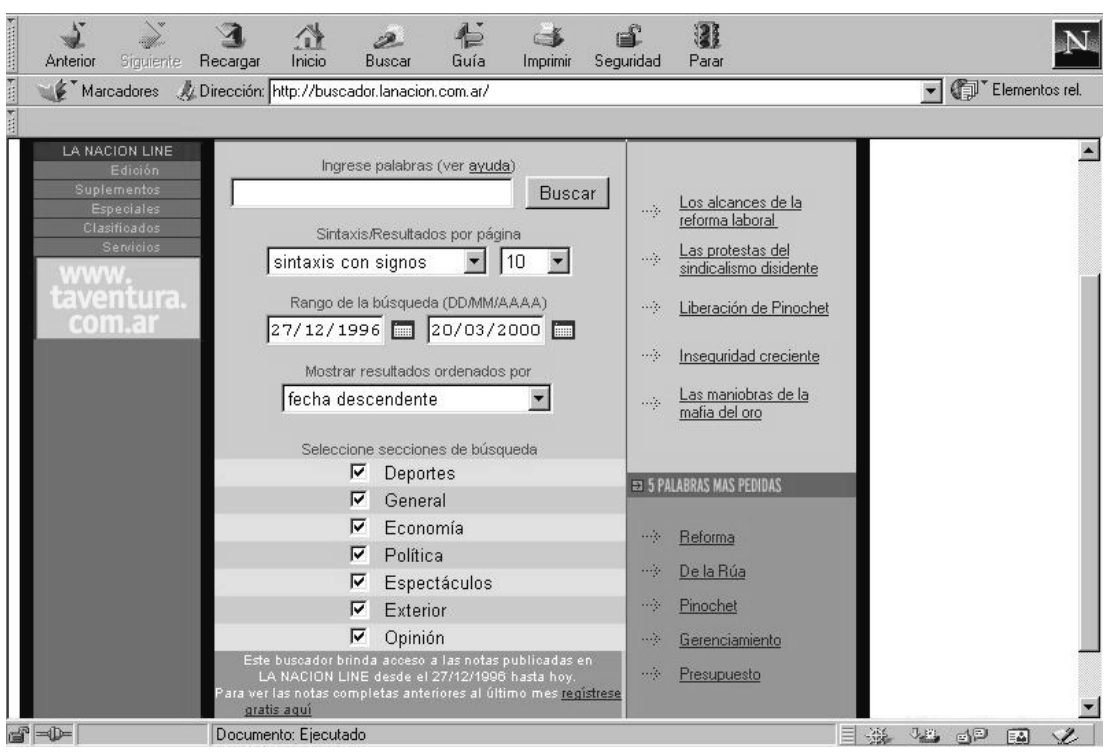

Formulario de búsqueda de La Nación

necesidades de los usuarios, ya sean los propios periodistas, investigadores o público en general.

Las hemerotecas en línea son uno de estos servicios de valor añadido y son la base de este artículo. En él no se pretende realizar un trabajo exhaustivo de análisis, sino una presentación del estado de la cuestión con una valoración de las hemerotecas digitales de los rotativos que tienen, además de la edición impresa, una versión electrónica. Por ello, y dado que no es el objetivo de este trabajo, no se hablará de los diarios en formato exclusivamente digital.

\section{«Entre los diez títulos que en la actualidad ofrecen una mayor cobertura cronológica en sus archivos sólo uno, Le Monde, es europeo»}

\section{Sistemas de acceso a la información retrospectiva}

Las hemerotecas de los diarios en internet suelen adoptar en la actualidad dos formas de presentación: una en la que se ofrece al usuario la posibilidad de hojear ejemplares retrospectivos íntegros (browsing ${ }^{1}$ ), tal y como lo haría en una hemeroteca tradicional, y otra en la que se constituye un archivo digital mediante la creación de una base de datos documental alimentada a través del volcado de contenidos de la edición electrónica.

1. Browsing de ejemplares publicados. Fue uno de los primeros servicios documentales de valor añadido proporcionados por la prensa en internet mediante el cual el lector puede acceder al número que desea especificando la fecha en que fue publicado. El acceso al ejemplar suele ser, en la mayoría de los diarios, gratuito.

Como refleja la tabla 1, es una opción muy presente entre los grandes rotativos europeos e hispanoamericanos, a diferencia de lo que ocurre con los diarios norteamericanos que se han decantado por ofrecer un tipo de acceso a sus hemerotecas mediante la creación de bases de datos documentales.

Al analizar los datos relativos a España (tabla 2) destaca la ausencia de algunos grandes diarios de información general como $A B C$ o $E l$ País, que disponen de un servicio de hemeroteca testimonial que incluye únicamente los ejemplares de la última semana.
La cobertura geográfica de los diarios analizados ha sido mundial —fundamentalmente norteamericanos, sudamericanos y europeos, haciendo un énfasis especial en los españoles-y se han escogido los que poseen las hemerotecas digitales más sofisticadas, con los servicios más completos y las mejor elaboradas. Ello ha sido posible gracias a la línea de investigación que se sigue desde hace ya algún tiempo en la $F a$ cultad de ciencias de la comunicación, Área de documentación, de la Universidad Autónoma de Barcelona.

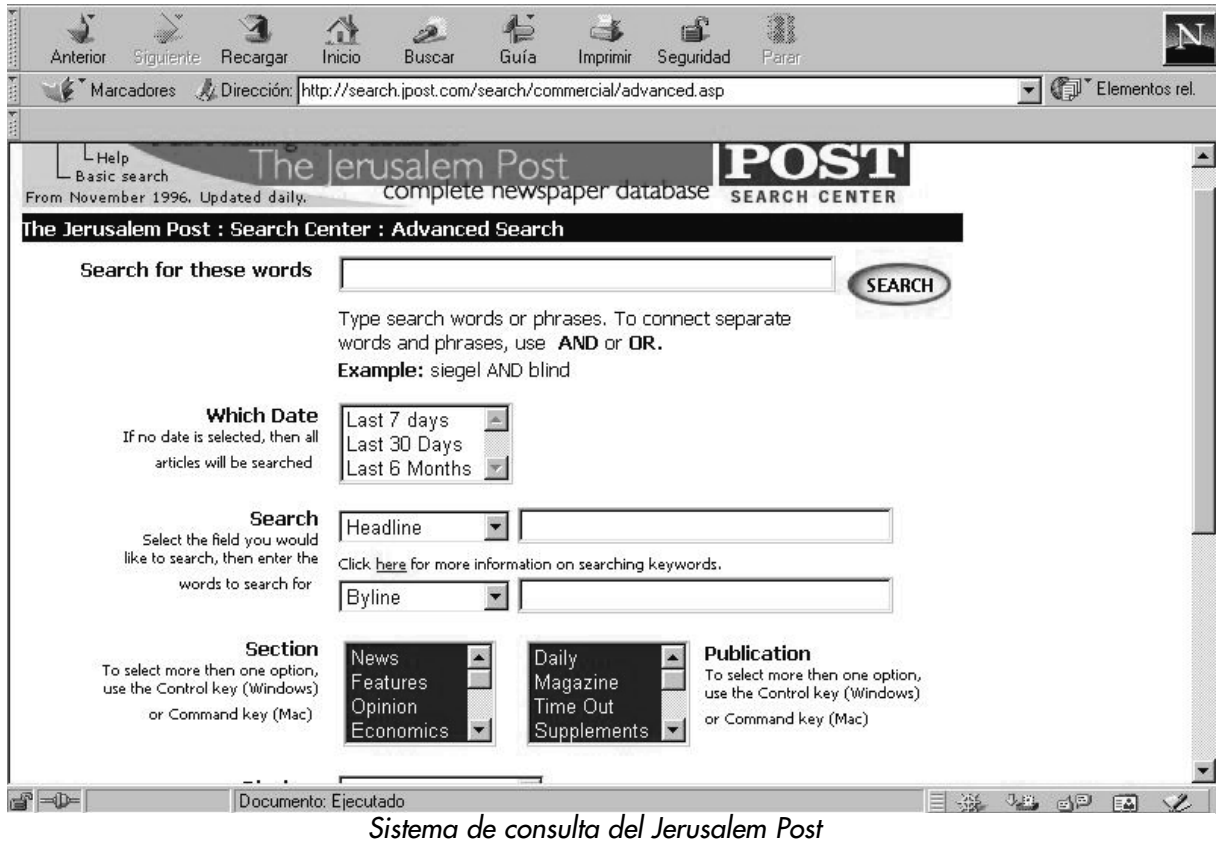




\section{Consulta de hemerote- cas a través de bases de datos.} La oportunidad que brinda la Red a los diarios para trasformarlos en potentes fuentes de información retrospectiva se materializa a través de los sistemas de interrogación de sus archivos. Este servicio va mucho más allá de la simple consulta del ejemplar concreto y permite explotar de forma selectiva grandes volúmenes de información gestionados a través de bases de datos documentales. Según hemos dicho, los diarios norteamericanos se han inclinado por este tipo de sistema de acceso a sus hemerotecas. Como muestra la tabla 3 , entre los diez títulos que en la actualidad ofrecen una mayor cobertura cronológica en sus archivos sólo uno, Le Monde, es europeo, lo que corrobora la idea de que Europa y el resto del mundo tienen todavía un considerable camino por recorrer en lo que se refiere al desarrollo de este tipo de servicios que, sin duda, proporcionan unas posibilidades mucho más ricas de explotación de la información retrospectiva.

Es significativa la diferencia en cuanto a la cobertura cronológica de los archivos de los diarios norteamericanos en relación con los principales medios europeos e hispanoamericanos (ver tabla 4).

El acceso a este tipo de archivos no es siempre gratuito. La cantidad de información disponible y la calidad de los recursos técnicos para su explotación expli-

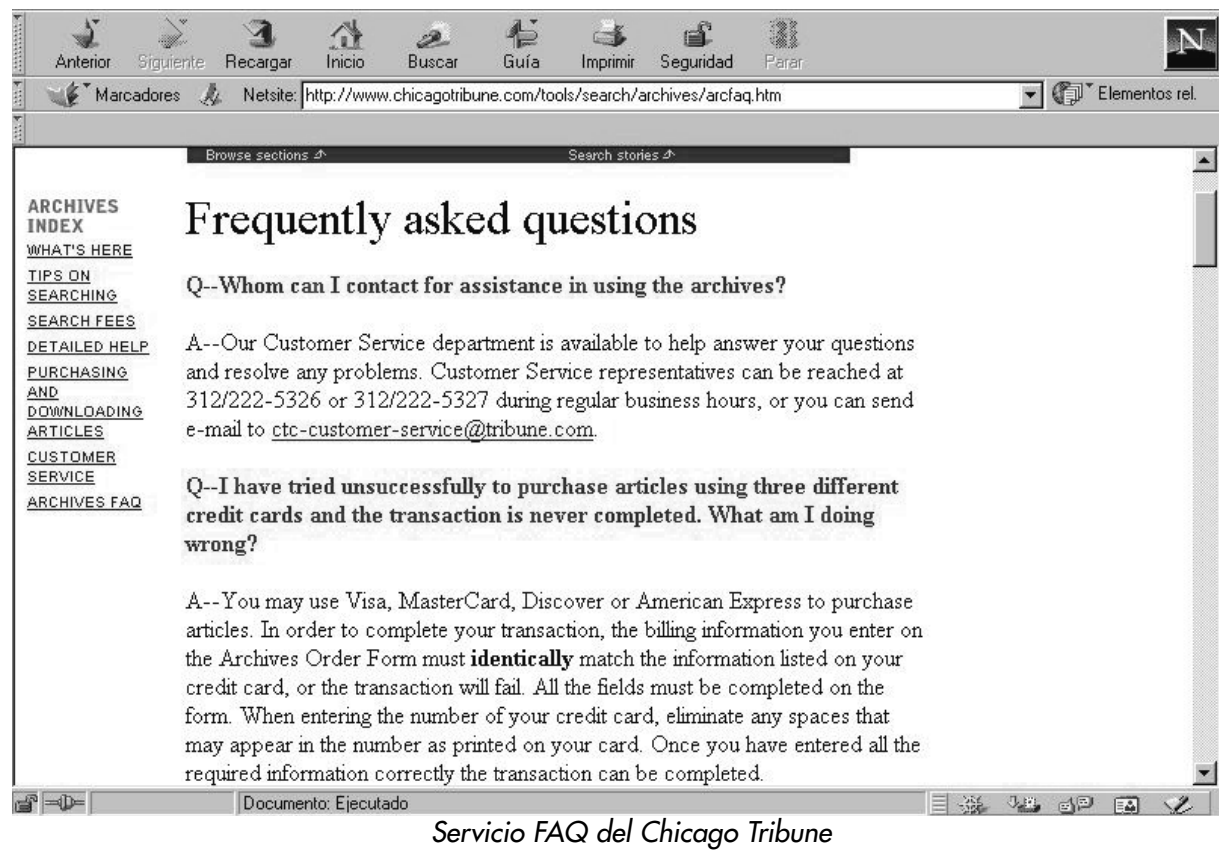

can las razones de su gratuidad o no. En la gran mayoría de los diarios estadounidenses el acceso a la información a través de su archivo es de pago, si bien la consulta es siempre gratuita. En Europa, rotativos como el alemán Die Welt o el portugués Jornal de Notícias, con archivos realmente ricos, ofrecen el acceso a su hemeroteca de forma gratuita. En cambio, la consulta de los archivos de los rusos Moscow Times y St. Petersburg Times exige una subscripción de pago al servicio. También Il Corriere della Sera sólo es accesible mediante este sistema, aunque la consulta de las informaciones publicadas en la última semana sea gratuita.

Le Monde es quizá el caso más peculiar. Como sucede en los títulos norteamericanos, la búsqueda de información en el archivo no tiene cargos pero el acceso a la noticia sí. La peculiaridad estriba en el coste del artículo ya que éste depende de su extensión. Así, la consulta de informaciones breves, no superiores a los 400 caracteres es libre, mientras que el coste del resto de noticias varía en función de su longitud: existen artículos cortos (hasta 1.500 caracteres), medios (de 1.500 a 5.000) y largos (superiores a $5.000)$.

En España, la búsqueda y el acceso a noticias en la treintena de hemerotecas digitales disponibles en la actualidad es, en todos los diarios, gratuita. Como se muestra en la tabla 5, los archivos digitales españoles que incluyen mayor volumen de in- 
formación retrospectiva corresponden a dos diarios especializados: Diario Médico y el económico Expansión. También es significativa la importancia concedida a estos servicios por parte de títulos locales como Levante, Segre o el Diario de Ibiza.

\section{Tipos de información accesible.}

- Información textual. En la información accesible a través de las diversas formas de presentación que adoptan las hemerotecas suelen excluirse generalmente tanto los datos meteorológicos como los anuncios clasificados, para los que en algunos rotativos norteamericanos existen sistemas de búsqueda especiales.

En otros casos, la información retrospectiva disponible se centra únicamente en los despachos de agencia, como sucede en el Moroccan Today. Jerusalem Post, Clarín y La Nación, por otro lado, además de las ediciones del diario proporcionan acceso a sus suplementos semanales. El alemán Hamburger Morgenpost cuenta también con una base de datos que incluye las críticas cinematográficas en él publicadas. De forma similar El País añade un sistema de consulta de las reseñas sobre libros, películas y productos multimedia incluidas en su suplemento Tentaciones.

- Información gráfica. Son escasos los diarios que ofrecen la posibilidad de buscar y recuperar aquella publicada con anterioridad. San Francisco Chronicle es el único rotativo que, de forma gratuita, ha puesto a disposición de sus lectores el material gráfico aparecido desde 1997 a través de un refinado sistema de búsqueda. Otros diarios norteamericanos como Usa Today o New York Times remiten a sus respectivos servicios de documentación para la adquisición de este tipo de información. Lejos del contexto norteamericano, en Australia se encuentra una interesante iniciativa para ofrecer acceso al material gráfico publicado en los principales medios del país llamado Newspix y, aunque se trata de un servicio de pago, la búsqueda y vi-

\begin{tabular}{|l|l|}
\hline \multicolumn{1}{|c|}{ Publicación } & \multicolumn{1}{c|}{ Cobertura } \\
\hline Diario Médico & 1997 (feb.) \\
\hline El Mundo & 1998 (ene.) \\
\hline Sport & 1998 (ene.) \\
\hline Expansión & 1998 (abr.) \\
\hline El Periódico & 1999 (jul.) \\
\hline El Día de las Baleares & 1999 (ago.) \\
\hline As & 6 meses \\
\hline Avui & 5 meses \\
\hline La Vanguardia & 5 meses \\
\hline El Mundo Deportivo & 4 meses \\
\hline Correo Español & 1 mes \\
\hline
\end{tabular}

Tabla 2. Diarios españoles con mayor cobertura en su servicio de browsing

\begin{tabular}{|l|l|l|l|}
\hline \multicolumn{1}{|c|}{ Publicación } & \multicolumn{1}{c|}{ Cobertura } & \multicolumn{1}{c|}{ País } & \multicolumn{1}{c|}{ Servicio } \\
\hline Sant Petersburg Times & 1994 (jul.) & Rusia & Pago \\
\hline San Francisco Chronicle & 1995 (ene.) & EUA & Gratuito \\
\hline Die Welt & 1995 (may.) & Alemania & Gratuito \\
\hline La Nación & 1995 (dic.) & Argentina & Gratuito \\
\hline The Times & 1996 (ene.) & Reino Unido & Gratuito \\
\hline Clarin & 1996 (mar.) & Argentina & Gratuito \\
\hline El Universal & 1996 (jun.) & Venezuela & Gratuito \\
\hline Globes & 1996 (jul.) & Israel & Gratuito \\
\hline L'Humanité & 1996 (ago.) & Francia & Gratuito \\
\hline Hindustan Times & 1996 (dic.) & India & Gratuito \\
\hline Morrocan Today & 1997 (sep.) & Marruecos & Gratuito \\
\hline El Observador & 1998 (jun.) & Uruguay & Gratuito \\
\hline Jerusalem Post & 1999 (mar.) & Israel & Gratuito \\
\hline
\end{tabular}

Tabla 1. Diarios internacionales con mayor cobertura en su servicio de browsing

sualización de las imágenes es totalmente gratuita. Incluye información aparecida desde 1998.

4. Archivos de grupos editoriales. Otra interesante iniciativa en relación a las hemerotecas digitales es la llevada a cabo por algunas empresas editoriales que distribuyen varios diarios. Consiste en la creación de un método de consulta común que posibilita búsquedas simultaneas en más de un periódico. Así, es posible interrogar a la vez los archivos de los británicos The Guardian y The Observer mediante un único sistema. En este sentido cabe mencionar también Globnet, un servicio que permite consultar los archivos de los medios del grupo RCS que incluye Il Corriere della Sera y La Gazzetta dello Sport, con una hemeroteca que arranca en enero de 1996 (la mayor de entre los diarios deportivos europeos).

\section{«En España, la búsqueda y el acceso a noticias en la treintena de hemerotecas digitales dispo- nibles en la actualidad es gra- tuita»}

En España no hay iniciativas similares pese a la existencia de grupos editoriales con una fuerte presencia de diarios en la Red. El caso más significativo lo constituye el Grupo Correo con cerca de una decena de cabeceras que disponen de una pequeña hemeroteca digital.

\section{Otras iniciativas de acceso a hemerotecas di-} gitales. Al margen de aquellas de diarios específicos o de grupos editoriales, se han puesto en marcha otros proyectos de creación de archivos para dar acceso a información retrospectiva. Así, por ejemplo, en Australia existe un servicio gratuito que agrupa las noticias publicadas desde inicios del 2000 en los principales diarios del país.

Financial Times, desde la página de su archivo, permite al lector seleccionar en qué medios efectuar su búsqueda, pudiendo limitarla a los últimos tres años del diario o ampliarla a la información económica apa- 


\begin{tabular}{|l|c|l|l|}
\hline Publicación & Cobertura & País & Servicio \\
\hline The Washington Post & 1977 & EUA & Pago \\
\hline Philadelphia Daily News & 1978 & EUA & Pago \\
\hline The Boston Globe & 1979 & EUA & Pago \\
\hline Philadelphia Inquirer & 1981 & EUA & Pago \\
\hline El Nuevo Herald & 1982 & EUA & Pago \\
\hline Miami Herald & 1982 & EUA & Pago \\
\hline Detroit Free Press & 1982 & EUA & Pago \\
\hline Chicago Tribune & 1985 & EUA & Pago \\
\hline San Jose Mercury & 1985 & EUA & Pago \\
\hline Le Monde & 1987 & Francia & Pago \\
\hline
\end{tabular}

Tabla 3. Los diez diarios internacionales con mayor cobertura en sus archivos digitales

recida en los últimos seis meses de una selección de los principales diarios internacionales. Existe una tercera opción que amplía la consulta a una colección de cerca de 3.000 publicaciones de todo el mundo.

En este terreno los diarios norteamericanos llevan la delantera al resto del mundo. Newslibrary es un archivo de noticias que agrupa las informaciones aparecidas en más de 80 rotativos estadounidenses y canadienses. Es un servicio de pago, aunque la búsqueda sea gratuita. El sistema de consulta es altamente refinado. El volumen de la información contenida en los archivos varía en cada diario, aunque en muchos casos su cobertura temporal se inicia a mediados de la década de los 80. Newslibrary incluye mayoritariamente archivos de diarios locales, aunque cuenta con otros de prestigio internacional como Washington Post, Boston Globe o San Jose Mercury. Muy interesante es también el servicio de pago Electric library, actualizado

\begin{tabular}{|l|c|l|}
\hline Publicación & Cobertura & Servicio \\
\hline Le Monde & 1987 & Pago \\
\hline Les Echos & 1991 & Gratuito \\
\hline Corriere della Sera & 1992 & Pago \\
\hline Moscow Times & 1994 & Pago \\
\hline St. Petersburg Times & 1994 & Pago \\
\hline Die Welt & 1994 & Gratuito \\
\hline Jornal de Noticias & 1995 & Gratuito \\
\hline De Telegraaf & 1996 & Gratuito \\
\hline Frankfurten Allgemeine & 1996 & Pago \\
\hline Hamburger Morgenpost & 1996 & Gratuito \\
\hline L'Humanité & 1996 & Gratuito \\
\hline La Nación & 1996 & Gratuito \\
\hline Clarin & 1997 & Gratuito \\
\hline O Globo & 1997 & Gratuito \\
\hline Financial Times & 1997 & Gratuito \\
\hline
\end{tabular}

Tabla 4. Diarios europeos e hispanoamericanos con mayor cobertura en sus archivos digitales

diariamente, que ofrece la posibilidad de efectuar búsquedas simultáneas en 150 diarios en su mayoría de ámbito anglosajón, cientos de revistas, noticias de agencias internacionales, mapas y material gráfico.

\section{Cómo consultar las hemerotecas}

En este apartado se van a describir las características principales que presentan los archivos soportados por un sistema de gestión de bases de datos. Cada vez es más habitual encontrar que los diarios proporcionen dos sistemas de búsqueda a sus lectores. Uno simple para peticiones de información sencillas y otro más avanzado para efectuar consultas con un nivel mayor de complejidad. De forma incipiente algunos rotativos,

\title{
Próximos números monográficos
}

\author{
Septiembre 2000 Consultores en recursos de información
}

\author{
Diciembre 2000 Información en ciencias de la salud
}

Los interesados pueden remitir notas, artículos, propuestas, publicidad, comentarios, etc., sobre estos temas a:

epi@sarenet.es 


\begin{tabular}{|l|l|}
\hline \multicolumn{1}{|c|}{ Publicación } & \multicolumn{1}{c|}{ Cobertura } \\
\hline Diario médico & 1997 (feb.) \\
\hline Expansión & 1998 (abr.) \\
\hline Levante & 1998 (may.) \\
\hline Segre & 1998 (jun.) \\
\hline Diario de Ibiza & 1998 (nov.) \\
\hline La voz de Almeria & 1999 (mar.) \\
\hline El Correo Gallego & 1999 (may.) \\
\hline El Dia de las Baleares & 1999 (ago.) \\
\hline La Vanguardia & 6 meses \\
\hline
\end{tabular}

Tabla 5. Diarios españoles con mayor cobertura en sus archivos digitales

como por ejemplo el argentino Clarín o el británico Financial Times, incluyen además en su formulario de consulta la opción de efectuarla en su hemeroteca o ampliarla a toda la Red, así como la posibilidad de guardar la estrategia de búsqueda para reutilizarla posteriormente.

A la hora de llevarla a cabo, el usuario selecciona los términos que considera más apropiados utilizando el lenguaje natural, por lo que la precisión de la consulta está condicionada por los problemas derivados del uso de este procedimiento (polisemia, ambigüedad, etc.). La utilización del lenguaje natural se explica por la ausencia de análisis documental de las informaciones que forman parte de las hemerotecas en la mayoría de los diarios. Para mejorar la precisión en la búsqueda y recuperación, en algunos casos se ha recurrido a diversas opciones: el sudafricano The Independent, una vez efectuada la búsqueda, elabora una lista con los cinco términos más significativos de cada documento recuperado. Este sistema no sólo permite conocer el nivel de precisión de la recuperación sino que ofrece también un método para refinar la consulta. Le Monde y Les Echos posibilitan limitar al tipo de artículo; este último

diario también clasifica las noticias de acuerdo con unas listas predefinidas de materias y ámbitos geográficos que el usuario puede utilizar a la hora de precisar su consulta. Lo mismo sucede en la revista Le Monde Diplomatique. Los Angeles Times es el único que admite buscar a partir de los descriptores que asigna su departamento de documentación.

1. Sintaxis de interrogación. El elemento fundamental que permite aprovechar la riqueza informativa de la hemeroteca de un diario en internet es el sistema que la gestiona, ya que determina las posibilidades de las que se dispone para interrogarla. En la tabla 6 se muestran los diarios que cuentan con un mayor número de recursos para la consulta de sus archivos.

2. Campos de búsqueda. Nos referimos en este punto a una de las posibilidades que los diarios ofrecen (ver tabla 7) para acotar la consulta a sus hemerotecas. Las opciones más usuales son: búsqueda por sección del diario, período cronológico y palabras contenidas en el titular. La limitación por sección, pese a estar presente en muchos casos, no es idéntica en todos ellos. Así, por ejemplo, en el español $A B C$ sólo es posible elegir una de las secciones para efectuar la búsqueda, mientras que rotativos con otros sistemas más depurados permiten realizarlas simultáneamente en más de una, como sucede con Jerusalem Post o Clarín.

\section{«Algunos rotativos incluyen en su formulario de consulta la op- ción de efectuarla en su heme- roteca o ampliarla a toda la Red, así como la posibilidad de guar- dar la estrategia de búsqueda»}

Por lo que respecta a la búsqueda por períodos cronológicos existen dos modalidades: que el lector pueda establecer libremente un rango cronológico (de la fecha A a la B), o bien, que deba elegir entre unos períodos predeterminados en el formulario de consulta: una semana, un mes, un año, todo el archivo, etc. Un caso singular lo constituye el brasileño $O$ Globo, que actualiza su edición constantemente y es el único que permite, entre sus opciones predeterminadas, efectuar búsquedas en las noticias recibidas en la última hora.

\begin{tabular}{|c|c|c|c|c|c|}
\hline Publicación & Booleanos & \begin{tabular}{|l|} 
Proximidad \\
\end{tabular} & Adyacencia & \begin{tabular}{|l|} 
Truncamiento \\
\end{tabular} & Paréntesis \\
\hline New York Times & $\bullet$ & & $\bullet$ & $\bullet$ & \begin{tabular}{|c|}
$\bullet$ \\
\end{tabular} \\
\hline Le Monde & $\bullet$ & $\bullet$ & $\bullet$ & & \\
\hline Liberation & $\bullet$ & $\bullet$ & & $\bullet$ & \\
\hline San Francisco Chronicle & $\bullet$ & & $\bullet$ & $\bullet$ & \\
\hline Moscow Times / St. Peters & $\bullet$ & $\bullet$ & & $\bullet$ & \\
\hline Corriere della Sera & $\bullet$ & & & $\bullet$ & $\bullet$ \\
\hline$A B C$ & $\bullet$ & $\bullet$ & $\bullet$ & & \\
\hline O Globo & $\bullet$ & & $\bullet$ & $\bullet$ & \\
\hline Chicago Tribune & $\bullet$ & & $\bullet$ & & \\
\hline Los Angeles Times & $\bullet$ & & & $\bullet$ & \\
\hline Die Welt & $\bullet$ & & & $\bullet$ & \\
\hline La Vanguardia & $\bullet$ & & $\bullet$ & & \\
\hline La Nación & $\bullet$ & & $\bullet$ & & \\
\hline Washington Post & $\bullet$ & & & & \\
\hline Financial Times & $\bullet$ & & & & \\
\hline
\end{tabular}

Tabla 6. Diarios que ofrecen mayor riqueza de recursos para la consulta de sus hemerotecas 
INTEGRIDAD DE I X F O MACION

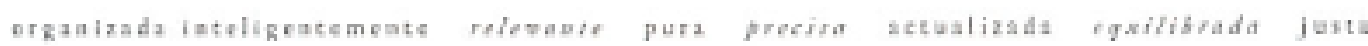

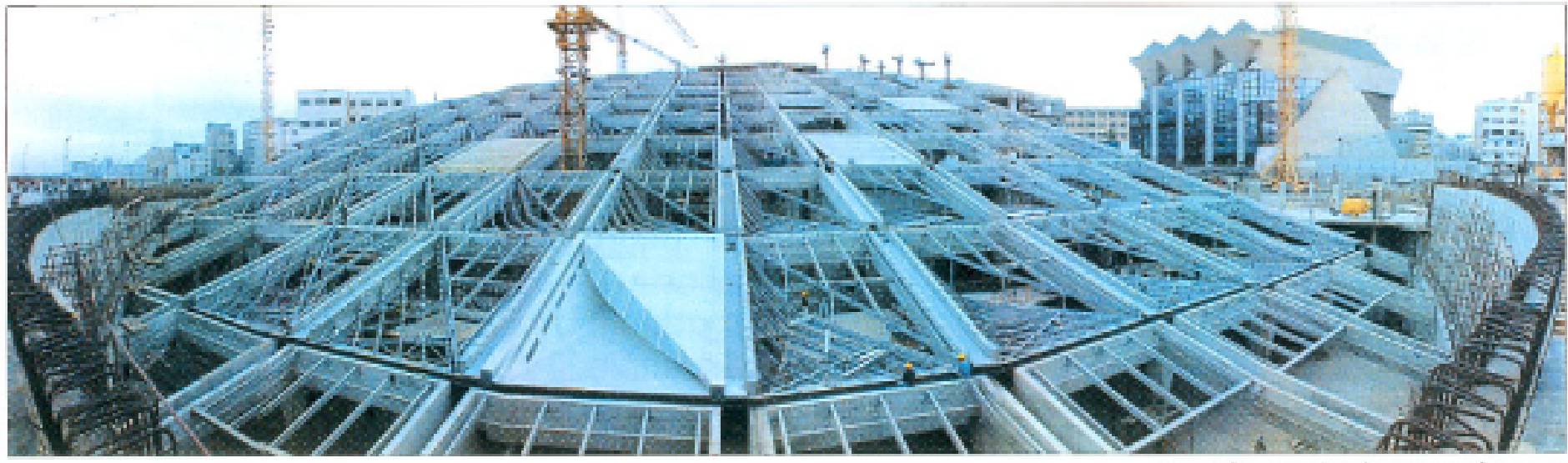

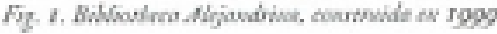

\section{Usted nunca tuvo un mejor momento para}

\section{CONSTRUIR SU PROPIO MONUMENTO A LOS CONOCIMIENTOS}

Con sus 700000 pergaminos, la biblioteca de Alciandria fue d mayor monumento a los conocimientos en d mundo encero. Fsta biblioseca, que fue destruida cerca del xîo 48 AC, fue nesucitada con el nocrabre de Bibliotheca Alejandrina. Afortunadamente, ya no se utilizan los pergaminos.

\section{PRESENTANDO INFOTRAC WEB}

Ahoca, combinando te Internet con b fabilidad de Gale Group, usted puede construir sta propio monumento a los conocimientos utilizando InfoThe Web. Esta revolucionaria interfix para referencias proporciona accoso a una gama extensa de bases de datos en texto completo de Gale Group. Se ofrece:

- Una interfaz que usted puede personalizar a su gusto

- Acreso remoto seguro para los usuarios registrados

- Búsquedas por materias y referencias de la más alta tecnoblogia
- Múltiples opciones de recuperación y entrega

- Resultados de búsqueda de acuerubo al rango de relevancia

- Grificas e imágenes

- Informes detallados de utilización en una variedad de formatos

\section{Y SOLAMENTE INFOTRAC WEB}

\section{OFRECE INFOMARKS}

Esta utilidad integral les permite a los usuarios recortar $y$ pegar bisquedas o enlaces de documentos de Info'Trac en páginas Web, e-mail y documentos de procesamicnto de pa bbras para crear listados de lectura, guáas de estudio y mis.

\section{INFOTRAC WEB SE OBTIENE GRATIS}

Y lo mejor de todo: casando compra cualquier base de datos InfoTrac used recile InfoTrac Web graris. Para recibir mis informacoxín contacte a su representante de Gale Group al internationalegalegroup.com.

INFOTRAC WEB

Interfaz que se parde personaliaar Contenido muy preniado Caliad de Gale Gronp 


\begin{tabular}{|l|c|c|c|c|c|c|c|c|c|}
\hline \multicolumn{1}{|c|}{ Publicación } & Sección & $\begin{array}{c}\text { Tipo de } \\
\text { articulo }\end{array}$ & Titular & Autor & Fecha & Lead & Subtítulo & $\begin{array}{c}\text { Palabra } \\
\text { clave }\end{array}$ & Tamaño \\
\hline Le Monde & $\bullet$ & $\bullet$ & $\bullet$ & $\bullet$ & $\bullet$ & & & & $\bullet$ \\
\hline Les Echos & $\bullet$ & $\bullet$ & $\bullet$ & $\bullet$ & $\bullet$ & & & $\bullet$ & \\
\hline San Francisco Chron. & $\bullet$ & & & & $\bullet$ & $\bullet$ & $\bullet$ & $\bullet$ & \\
\hline Jerusalem Post & $\bullet$ & & $\bullet$ & & $\bullet$ & & $\bullet$ & & \\
\hline Clarin & $\bullet$ & & $\bullet$ & & $\bullet$ & & $\bullet$ & & \\
\hline Washington Post & & & $\bullet$ & $\bullet$ & & $\bullet$ & & & \\
\hline Corriere della Sera & & & $\bullet$ & $\bullet$ & $\bullet$ & & & & \\
\hline ABC & $\bullet$ & & & & $\bullet$ & & & & \\
\hline OGlobo & $\bullet$ & & & & $\bullet$ & & & & \\
\hline La Nación & $\bullet$ & & & & $\bullet$ & & & & \\
\hline Levante & & & $\bullet$ & & $\bullet$ & & & & \\
\hline Financial Times & & & $\bullet$ & & $\bullet$ & & & & \\
\hline La Repubblica & $\bullet$ & & $\bullet$ & & & & & & \\
\hline New York Times & & & & & $\bullet$ & & & & \\
\hline Los Angeles Times & & & & & & & & $\bullet$ & \\
\hline The Times & $\bullet$ & & & & & & & & \\
\hline
\end{tabular}

Tabla 7. Diarios que cuentan con mayor variedad de campos de búsqueda

No todos los diarios contemplan ambas modalidades, como en el caso de Le Monde.

Algunos sistemas de gestión documental cuentan con la posibilidad de buscar por elementos que conforman la noticia: titular, subtítulos, autor o lead (entrada o primer párrafo con los datos básicos). Clarín o San Francisco Chronicle incluyen, por ejemplo, la opción de buscar informaciones a partir de los términos que aparecen en el lead o en los subtítulos. Le Monde facilita la posibilidad de determinar la dimensión del documento a recuperar ya que, como se ha dicho, de ello depende el coste de la consulta a su hemeroteca.

3. Visualización de resultados. En sus formularios de búsqueda los principales diarios internacionales permiten determinar ciertos parámetros a la hora de ver en pantalla los documentos recuperados: número máximo a visualizar, modo de presentación —una forma simple que muestre sólo el titular de la noticia o bien opciones más detalladas que pueden incluir: subtítulos, sección, fecha, autor y lead como en el caso de Chicago Tribune- También es posible definir el criterio de ordenación de los documentos: cronológico o ponderado. Este último depende de un algoritmo que suele valorar el nivel de relevancia del documento de acuerdo con el número de ocurrencias de los términos solicitados en la búsqueda combinado con su ubicación en el texto.

El volumen de información de cada documento que se proporciona puede actuar como un valioso indicador para conocer el nivel de precisión y adecuación de los resultados obtenidos. Así, existen indicaciones auxiliares que permiten contextualizar y valorar el interés del documento como: subtítulos, sección, autor o lead. A través de estos elementos el lector puede seleccionar mejor la noticia que quiere visualizar de forma íntegra. Les Echos y Clarín señalan incluso si las que han sido recuperadas se acompañan de información gráfica o no. El recurso de destacar los términos de búsqueda utilizados en los documentos obtenidos, como hacen Clarín o The Times, constituye también un buen ejemplo de la utilidad e importancia de este método. Una función similar la cumple la información sobre el número de ocurrencias de los términos de búsqueda en los documentos rescatados, sistema utilizado por el San Jose Mercury.

\section{«Las hemerotecas digitales se están configurando como uno de los más interesantes servi- cios de valor añadido propor- cionados por los diarios en in- ternet»}

En la tabla 8 se recogen las indicaciones auxiliares más usuales proporcionadas por los principales diarios internacionales.

4. Ayudas en línea. La inmensa mayoría de los diarios cuenta en la actualidad con páginas de ayuda para optimizar el proceso de consulta de su hemeroteca. En algunos de los españoles este tipo de servicio se ha incorporado con lentitud y continúa siendo en ciertos casos poco explicativo para obtener un buen aprovechamiento de las prestaciones de sus sistemas de búsqueda. Así, por ejemplo, casi nunca existen referencias a la sensibilidad de ciertos caracteres como cifras, acentos o mayúsculas. Entre los diarios extranjeros es usual que sus páginas de ayuda sean en realidad un servicio $F A Q$ que oriente al usuario en todo lo 
relacionado con la consulta de la hemeroteca digital: su precio, cobertura del archivo, tipo de información disponible, etc. En otros casos estas mismas páginas informan sobre cómo acceder a las ediciones no disponibles en dicha hemeroteca: Los Angeles Times da noticia de su hemeroteca microfilmada; The New York Times remite desde su web a otros servicios de información como bases de datos y bibliotecas públicas donde consultar el diario; $O$ Globo publicita un servicio de búsqueda en los archivos microfilmados de los principales diarios de Río y São Paulo desde 1925 y Le Figaro, Liberation y Le Parisien, con hemerotecas testimoniales en la Red, remiten al servicio Minitel.

\section{Dosieres temáticos}

Existe entre los principales rotativos europeos una tendencia a crear dosieres sobre temas de interés prolongado en los que se recogen las principales informaciones publicadas sobre cada uno de ellos. Los mejores ejemplos los constituyen los franceses Le Monde, Libération, L'Humanité, así como el portugués Jornal de Notícias y los británicos The Times y The Guardian. En España destacan La Vanguardia y El País. The Wall Street Journal dispone de detallados dosieres de información sobre empresas.

\section{Hacia la hemeroteca personalizada}

La recepción vía e-mail permite en algunos casos una personalización del diario y, en definitiva a través de una definición más ajustada de las necesidades informativas del lector, elaborar dosieres temáticos que constituyan también archivos o hemerotecas personalizadas. Evidentemente no todos los diarios que ofrecen la posibilidad de remitir a sus lectores la edición electrónica tienen las mismas características.

De entre los más de 50 diarios españoles presentes en internet actualmente son 7 los que ofrecen gratuitamente un servicio de recepción de titulares: La Vanguardia, Canarias 7, Diario Vasco, Diario de Navarra, Gaceta de los Negocios, Expansión, Diario Médico y El País. Este último es el único que permite seleccionar tanto las secciones de interés como los días de la semana que se desean recibir.

Fuera, Los Angeles Times y La Nación, por ejemplo, van un poco más allá: además de suministrar titulares de noticias permiten ser mucho más selectivos a la hora de especificar los contenidos. En el caso del diario californiano es posible seleccionar informaciones de hasta 10 ámbitos temáticos sobre una lista predefinida. La Nación, como La Vanguardia, también remite los titulares de los suplementos semanales que publica. Clarín envía gratuitamente una síntesis de los resultados deportivos.

Jerusalem Post, The New York Times y Usa Today, este último a través de su daily briefing, representan buenos ejemplos de rotativos que remiten el texto íntegro de una selección de noticias de las principales secciones. En todos los casos se trata de servicios gratuitos.

Los diarios más versátiles en este tipo de servicio son los econó-

Tabla 8. Diarios internacionales con una mayor gama de indicaciones auxiliares

1. Las abreviaturas utilizadas en los encabezamientos de esta tabla corresponden a: tit. = titular; subt. = subtítulo; secc. = sección; aut. = autor; $T$. dest. = término destacado; relev. = grado de relevancia 
micos. Financial Times, Les Echos y The Wall Street Journal permiten al lector editar de forma muy detallada su perfil de interés para la recepción de información. Les Echos cuenta con un completísimo servicio de alerta personalizada que, al margen de determinar los contenidos que se desean recibir, da opción para fijar la frecuencia de recepción. Otro tanto sucede con The Wall Street Journal, que ofrece una gama de posibilidades muy amplia para establecer los ámbitos de interés de sus lectores: secciones, índices bursátiles, datos sobre empresas, etc.

\section{A modo de valoración final}

Las hemerotecas digitales se están configurando como uno de los más interesantes servicios de valor añadido proporcionados por los diarios en internet. De los datos presentados en este artículo se desprende que los Estados Unidos se encuentran a la cabeza de los países con mayor número de diarios en la Red, así como que sus hemerotecas son las más desarrolladas tanto por su cobertura cronológica como por los recursos que tienen para su explotación. Europa y América Latina cuentan también con numerosas hemerotecas digitales y si bien el volumen de información consultable no es comparable al abarcado por los rotativos norteamericanos, en cambio sí son semejantes en cuanto a nivel de calidad. En España, de los más de cincuenta diarios con presencia digital, algo más de una treintena dispone de una hemeroteca aunque ninguna de ellas es equiparable a cualquiera de las analizadas y destacadas del resto de países, ni por su cobertura temporal ni por los recursos de búsqueda que contemplan. Llama la atención las enormes diferencias que se manifiestan entre las de los principales rotativos españoles de información general. Creemos que esta situación puede deberse a la fase transitoria por la que atraviesan las iniciativas de constitución de hemerotecas digitales en este tipo de diarios.

\section{«Electric library ofrece la posi- bilidad de efectuar búsquedas simultáneas en 150 diarios en su mayoría de ámbito anglosa- jón, cientos de revistas, noticias de agencias internacionales, mapas y material gráfico»}

Las tendencias que se observan en los diarios con hemerotecas de mayor riqueza informativa y mejor elaboradas son:

- El acceso a este servicio tiende a hacerse de pago, lo que contribuye a la calidad del mismo. Sin un producto competitivo no habrá usuarios que se subscriban.
- Se tiende a reemplazar la hemeroteca digital como fiel reflejo de la de carácter tradicional por archivos de información retrospectiva creados a partir de sistemas de gestión de bases de datos capaces de proporcionar noticias selectivas de entre grandes volúmenes de información. El sistema de consulta mediante browsing se sustituye por la búsqueda como base de datos.

- Existe un creciente interés por proporcionar opciones para perfeccionar y refinar las búsquedas y también por añadir valor a los resultados obtenidos para facilitar así la selección de aquellas noticias a las que se accederá en su formato íntegro.

\section{Notas}

1. Se ha utilizado el término browsing por ser el de uso más extendido para referirse a la consulta de hemerotecas ejemplar por ejemplar.

\section{Bibliografía}

Armañazas, Emy; Díaz Noci, Javier; Meso, Koldo. El periodismo electrónico. Información y servicios multimedia en la era del ciberespacio. Barcelona: Ariel, 1996.

Codina, Lluís. "Directorio selectivo de sedes web para documentalistas, estudiosos de los medios de comunicación social y profesionales de la información". En: El profesional de la información, 1997, septiembre, v. 6., n. 9 , pp. $32-38$.

Díaz Noci, Javier; Meso Ayerdi, Koldo. "Desarrollo del periodismo electrónico". En: El profesional de la Información, 1998, diciembre, v. 7, n. 12, pp. 4-11.

Díaz Noci, Javier; Meso Ayerdi, Koldo. Medios de comunicación en internet. Madrid: Anaya, 1997.

Fuentes i Pujol, Mª Eulàlia; González, Alfons. "La prensa española en internet. Análisis de los servicios de valor añadido". En: 6as. Jornadas españolas de documentación: los sistemas de información al servicio de la sociedad, 1998, pp. 281-292. Isbn 84-331-4609-X.

Fuentes i Pujol, Mª Eulàlia. La información en internet. Barcelona: Cims, 1997. Isbn 84-89643-31-8.

Jiménez, Angels; González, Alfons; Fuentes i Pujol, Mª Eulàlia. "Gestió documental de la informació en els serveis de valor afegit de la premsa espanyola a internet”. En: 7es. Jornades catalanes de documentació: les biblioteques i els centres de documentació al segle XXI: pea clau de la societat de la informació, 1999, pp. 405-417. Isbn 84-86972-9-4.

Marcos Recio, Juan Carlos. La documentación electrónica en los medios de comunicación. Madrid: Fragua, 1999. Isbn 84-7074-110-1.

Àngels Jiménez. Prof. ayudante, Àea de Documentació. Facultat de Ciècies de la Comunicació. UAB. ajimenez@kane.uab.es

Alfons González. Prof. asociado, Àea de Documentació. Facultat de Ciùcies de la Comunicació. UAB. alfgonz@teleline.es

M. Eulàlia Fuentes i Pujol. Catedrática de documentación, Àea de Documentació. Facultat de Cì̀cies de la Comunicació. $U A B$. eulalia.fuentes@uab.es 